Material Science

Opinion

Copyright (C) All rights are reserved by Leonard J Bond

\title{
Nondestructive Evaluation an Integral Part of Engineering
}

\author{
Leonard J Bond* \\ Aerospace Engineering, Iowa State University, USA
}

*Corresponding author: Leonard J Bond, Aerospace Engineering, Iowa State University, USA.

Received Date: March 15, 2019

Published Date: May 28, 2019

\section{Introduction}

Nondestructive testing and evaluation (NDT\&E) started as a tool to assess workmanship. Together with the related fields of condition-based maintenance and structural health it is recognized as central to ensuring initial product quality, as well as safety and reliability in service. NDT\&E is now seeing another metamorphosis: it is moving from being focused on assessing and ensuring its reliability and both detection and sizing of discrete defects to a more complete material state assessment. It is becoming an integral part of in-process measurement and QA/QC including a range of advanced processes and materials.

Since the 1950's nondestructive testing (NDT) has also become known as nondestructive evaluation (NDE) and it has been an area that has seen continued research, growth and evolution. It is an area closely linked to applications in aerospace engineering, civil engineering, electrical engineering, material science and engineering, mechanical engineering, nuclear engineering, petroleum engineering and based firmly on fundamental physics phenomena of energy-material interaction It is an interdisciplinary endeavor that is as essential to engineering practice as stress analysis, including for life estimation and prognostics.

The various NDT\&E measurement modalities, including using x-ray, ultrasound, electromagnetics as well as magnetic particles and penetrants have tended in large part to be relegated to be an after though, only applied at the end of a manufacturing process. Increased use of advanced manufacturing, including using composites, additively manufactured materials, ceramics and a diverse range glued and solid state joints need material characterization and microstructural level assessments, as well as parameters such as local, nondestructively measured, bond strength data, if new materials, design concepts and applications and to be fully utilized. There is no-longer an assumption of assuring freedom from defects, designs need to live with a defined acceptable population of and allowable anomalies, and parts need to be retired based on an assessed condition rather than at a nominal life.

There is a need to consider design for inspect ability, both for finished parts when using changing technologies including computed tomography and ultrasonic phased array combined with design application of NDE and stress analysis both using computer modeling. Inspection data then needs to be able to be analyzed in the context of a 3-D visualization and finite element analysis. There is also a need for new tools and techniques that reduce the unreliability of the human aspects of inspection and use both automation and robotics, with data analysis to provide capability for both real-time and remote inspection assessment, including using wireless technologies in the context of innovations such as Industry 4.0 .

The changes in the needed capabilities of NDE for material state assessment and analysis cannot just be met using a technician level activity with an ASNT or other entity certified inspector. There is a need for design and stress analysis engineers to have a better understanding of the capabilities and limitations of a multitude of NDE methods, including the statistics of performance measured using a probability of detection (POD) analysis. There is a need for at least some staff in manufacturing who can be an NDE Engineer. Outside the USA such engineers are now increasingly being seen.

In looking at the changing landscape as NDE become material state awareness (MSA) a fundamental question is how to meet the science and technology needs for advanced manufacturing and the staffing skill set given loss of experts through retirement and engineering workforce demographics. Not everyone in manufacturing needs to be an NDE expert, but a pipeline is need to provide experts for industry, researchers for the R\&D community in 
national laboratories and academia, who have the interdisciplinary skill set required to address critical quality challenges, provide insitu capabilities for advanced manufacturing and meet the needs of advanced QA/QC support for development and application engineers.

\section{Acknowledgement}

None.

\section{Conflict of Interest}

No conflict of interest. 